\section{$\underset{\substack{\text { hommes } \\ \text { \& migrations }}}{ }$}

\section{Hommes \& migrations}

Revue française de référence sur les dynamiques

migratoires

1319 | 2017

Réfugiés et migrants au Liban

\title{
Migrantes au Liban
}

L'église évangélique comme mode d'intégration sociale

\section{Fatiha Kaoues}

\section{OpenEdition \\ 1 Journals}

\section{Édition électronique}

URL : http://journals.openedition.org/hommesmigrations/3965

DOI : 10.4000/hommesmigrations.3965

ISSN : 2262-3353

\section{Éditeur}

Musée national de l'histoire de l'immigration

\section{Édition imprimée}

Date de publication : 1 octobre 2017

Pagination : 41-48

ISBN : 978-2-919040-39-1

ISSN : $1142-852 X$

Référence électronique

Fatiha Kaoues, « Migrantes au Liban », Hommes \& migrations [En ligne], 1319 | 2017, mis en ligne le 01 octobre 2020, consulté le 07 janvier 2021. URL : http://journals.openedition.org/hommesmigrations/ 3965 ; DOI : https://doi.org/10.4000/hommesmigrations.3965 


\title{
MIGRANTES AU LIBAN : L'ÉGLISE ÉVANGÉLIQUE COMME MODE D'INTÉGRATION SOCIALE
}

\author{
Par FATIHA KAOUES, chercheure postdoctorante, Groupe Sociétés, Religions, \\ Laïcités (Ephe-Sorbonne).
}

\begin{abstract}
Le Liban accueille des centaines de milliers d'étrangers, réfugiés ou migrants, qui constituent des cibles privilégiées pour les missionnaires évangéliques installés dans la région, profitant d'une réelle liberté d'expression dans ce pays. Les jeunes femmes migrantes employées en qualité de domestiques pâtissent de conditions d'exercice difficiles, aggravées par une législation défavorable aux étrangers. Dans ce contexte, l'intégration au sein d'un univers évangélique internationalement connecté offre des ressources pour améliorer leur statut aux plans individuel et collectif.
\end{abstract}

Le protestantisme au Liban est le fruit des missions américaines commencé au début du XIX ${ }^{\text {eiècle }}{ }^{1}$. La plupart des églises de type pentecôtiste se développe à l'est de la capitale. Parmi elles, Church of God s'engage particulièrement auprès des migrants et constitue l'espace privilégié de nos recherches². Pour saisir la place des migrants dans cette architecture protestante évangélique libanaise, il convient de rappeler que le Liban est un important pays d'accueil au Moyen-Orient pour les réfugiés de la région et les migrants du monde entier. Le pays est signataire de la plupart des traités de droits de l'Homme relatifs à la protection des réfugiés, à l'exception de la Convention de 1951. Dans ce contexte, les protestants évangéliques libanais qui accordent une importance majeure à la conversion voient dans cet afflux d'étrangers, des candidats possibles pour leur œuvre d'évangélisation. Cet article analyse le parcours de trois migrantes devenues chrétiennes évangéliques et met en évidence les potentialités qu'elles déploient mais aussi leurs limites dans le contexte d'une difficile évolution de leurs droits.

\section{Migrantes domestiques au Liban, un statut précaire}

Quelques données quantitatives empruntées à une grande étude statistique conduite par Ray Jureidini ${ }^{3}$ sur les travailleuses domestiques au Liban permettent d'appréhender au plus près le vécu de ces employées domestiques migrantes qui sont majoritairement originaires d'Éthiopie, des Philippines, du Bangladesh, de Sri Lanka, du Népal et de Madagascar. La grande majorité de ces femmes sont jeunes : $75 \%$ ont moins de 40 ans, Deux tiers d'entre elles travaillent onze heures par jour. Pour obtenir un emploi, elles paient des commissions très élevées aux agences de recrutement dans leur 
pays d'origine. Elles reversent aussi une commission à l'agence libanaise, ce qui contredit la réglementation promulguée par l'Organisation internationale du travail (OIT) ${ }^{4}$. Même si les conditions de travail sont très variables d'un employeur à l'autre, le seul fait de se voir confisquer presque systématiquement son passeport et de ne pouvoir sortir sans être accompagnée est en soi une servitude très lourde. Parmi les jeunes femmes interrogées, beaucoup se plaignent également de harcèlement verbal.

Les agressions physiques ou sexuelles sont le fait d'une minorité : elles concernent néanmoins un millier de personnes par an. Le système dit de la kafala (tutelle) installe une dépendance juridique à l'égard de l'employeur qui est en quelque sorte le garant; ce qui retire aux employées domestiques migrantes toute liberté de mouvement $t^{5}$. Exclues du droit du travail libanais, elles ne bénéficient, en effet, d'aucune protection légale. En dépit de ces faits accablants, le Liban demeure une destination attractive pour les migrants du fait de sa relative accessibilité et de son mode de vie « occidentalisé » vanté par les agences de placement. Dans ce pays, l'emploi de domestiques étrangères à domicile génère des millions de dollars de devises du fait de l'argent rapatrié par ces personnes vers leur pays d'origine. Un contrat type a été proposé en 2008 après concertation entre le ministère du Travail et diverses organisations non gouvernementales (ONG) libanaises très impliquées dans les droits des migrants, prévoyant des améliorations significatives de leurs conditions de travail. Cependant, le projet de loi n'est, à ce jour, toujours pas en vigueur.

\section{L'intégration sociale par l'église}

Au sein des églises évangéliques, les jeunes femmes étrangères constituent leurs propres groupes de prières. L'église Church of God (Kanîsât Allah) fondée en 2010 par Saïd Dib se mobilise particulièrement pour l'évangélisation des migrants. Installée dans la banlieue populaire de Burj Hammoud, l'église œuvre au « church planting " (zara' kanâyes), soit à la fondation d'églises qui est au cœur du travail d'évangélisation. À cet égard, le pasteur Dib revendique plusieurs milliers de convertis, de toutes nationalités, à lui seul. Dib tire fierté de ce cosmopolitisme qui atteste, à ses yeux, de la fraternité humaine, inséparable du projet évangélique. Il affirme ainsi son refus du nationalisme et considère que la foi évangélique est un lien plus précieux que celui de la nationalité. L'un des chants de son culte s'intitule "Yassou' aghla jensiyyé » ("Jésus est la "nationalité" la plus précieuse »). Au sein de l'église officie un groupe de prières constitué exclusivement de femmes migrantes, au nombre d'environ 80. Notre terrain, commencé en juin 2014 et achevé en décembre 2016, a été l'occasion d'une collecte de témoignages et d'une observation participante au sein de l'église. Sur les 14 entretiens approfondis que nous avons réalisés, nous en retenons ici trois. Ce choix se justifie du fait que les personnes en question rassemblent des caractéristiques spécifiques permettant d'analyser plusieurs aspects de ce développement évangélique en contexte migratoire.

Judy est une jeune femme malgache de 31 ans. Elle réside au Liban depuis 2010 et est employée au domicile d'une famille libanaise à Koreitem. Elle a essentiellement en charge la gestion courante du domicile, s'occupant des repas quotidiens et du ménage. Ses horaires de travail s'étendent de 7 heures 30 à 16 heures 30 environ. En matière de congé, la jeune femme dispose du samedi et du dimanche. Au sein de l'église, Judy a la responsabilité du «ministère musical ». Ayant appris à jouer de la guitare dans son pays d'origine, elle met ses talents au service du culte et accompagne les chants chrétiens de sa musique

Exaltaçion est une jeune femme de 27 ans originaire des Philippines. Elle est arrivée au Liban au début de l'année 2014. Employée à domicile, elle 
s'occupe d'un petit garçon dont la mère est directrice d'une école. En dehors du dimanche, la jeune femme ne dispose guère de temps libre et est censée être disponible à toute heure de la journée, de " 6 heures à minuit » confie-t-elle. Ses conditions de travail sont éprouvantes. Ses difficultés tiennent particulièrement dans sa faible autonomie. La jeune femme doit justifier le moindre de ses déplacements en journée, même pour une demi-heure. En dehors du dimanche, elle n'est pas autorisée à sortir.

Enfin, le cas d'Aimée est singulier : cette femme pasteur d'une cinquantaine d'année, originaire de Madagascar, est venue au Liban il y a une quinzaine d'années pour travailler en qualité d'employée à domicile. Elle s'est beaucoup impliquée au sein de l'univers religieux protestant. Désormais salariée par l'église, elle s'emploie à la fois à la défense des droits des migrantes mais aussi à leur évangélisation.

Bénéficiant de liens privilégiés avec des fidèles protestants expatriés employés dans les ambassades britannique et américaine, Aimée est parvenue à jouer de ce réseau diplomatique pour venir en aide aux migrantes en conflit avec leurs employeurs. Elle est ainsi devenue un intermédiaire reconnu aussi bien par les organisations en charge du recrutement de domestiques étrangères que par les ONG qui soutiennent ces jeunes femmes, comme Kafa (qui signifie littéralement « Ça suffit »).

\section{Conversions et reconstruction identitaire}

La plupart de ces femmes étaient croyantes, musulmanes ou de rite catholique avant leur conversion évangélique. L'islam ou le christianisme sont assimilés aux pratiques culturelles générales du pays de naissance. Le christianisme catholique ou l'islam prennent alors la forme d'un capital de mémoire, d'un patrimoine historique et culturel hérité plutôt que d'un véritable modèle de vie religieuse. La conversion s'effectue moins contre ces religions " héritées » que dans le cadre d'une absence de référents religieux définis en tant que tels.
Exaltaçion affirme que, même dans la famille libanaise qui l'accueille, la religion est très présente. Mais elle estime que cette religiosité (et les valeurs auxquelles elle est censée s'adosser) est contredite par l'attitude rarement compréhensive des patrons. "Ils ont tout le temps le nom de Dieu à la bouche mais c'est pas pour autant qu'ils sont sympas. Leur religion c'est comme une nationalité, il n'y a pas vraiment de place pour la foi. "C'est là un des arguments justifiant le choix du protestantisme évangélique, qui est le fruit d'une activité missionnaire, et donc «importé ». En effet, ce courant chrétien apparaît plus légitime pour la jeune femme car il ne figure pas au nombre des religions historiques qui sont, à ses yeux, discréditées par leur caractère politisé et communautarisé.

Dans son récit, Exaltaçion compare sans cesse le martyre de Jésus à sa situation personnelle, comme pour faire apparaître, par contraste, son quotidien moins oppressant. Toutes nos souffrances sont somme toute, aisées à relativiser comparées à celles endurées par Jésus pour sauver les hommes. "Le Christ s'est sacrifié pour nous. Il a pris notre propre fardeau pour nous en débarrasser. C'est un modèle pour nous tous, d'endurance et de bonté. "Cette leçon est un encouragement à la persévérance dans l'effort. Homme de voyage, il a subi la persécution de par son statut d' "étranger ", ce qui offre un parallèle marquant avec les migrantes.

Lorsque nous interrogeons Judy sur les raisons de son engagement dans l'église, elle évoque ses responsabilités au sein du «ministère musical ». «Je suis la responsable du ministère musical. Je dois préparer tous les chants du culte. Je fais ça avec Aymée et Ruth. On doit trouver les thèmes et je m'adapte à tous les répertoires. C'est très riche, c'est très beau. C'est une grande responsabilité. La Bible dit que les anges ont entendu la musique. La musique c'est très important dans le culte. On transmet tout l'amour du christ et des émotions sincères dans la musique. » Selon Judy, ses talents de musicienne trouvent leur pleine expression, du fait de son goût pour la guitare. Son amie Ruth, qui occupait dans son pays d'origine la fonction d'éducatrice dans un jardin d'enfant, prend en charge le ministère des enfants au sein de l'église. Le monde évangélique accorde ainsi un fort 
encadrement pour ces femmes. Ce qui marque, c'est la capacité de cet univers religieux à utiliser au mieux les potentiels du croyant: chacun de ses talents est investi au mieux de ses possibilités. C'est une foi qui s'exprime en acte. Le converti n'est pas passif, il intervient pleinement dans la vie religieuse; ce qui confère une valeur positive à son existence. De fait, le succès du
Originellement, la kafala constitue un concept juridique de droit coranique

qui désigne une mesure de recueil légal d'un enfant sans créer un lien de filiation, au contraire d'une adoption classique. protestantisme évangélique se justifie autant par sa plasticité que par sa capacité à apporter "des réponses expérientielles et morales au défi de la sécularisation moderne ${ }^{6}$.

Un aspect central qui intervient dans les récits de conversion porte sur le besoin de certitude. L'incertitude existentielle est le lot commun de nombreuses converties. Si l'islam ou le catholicisme ne confèrent apparemment pas de sentiment de certitude à propos du salut, le protestantisme évangélique insiste au contraire sur ce point. Aimée fait de cet aspect un point central de ses prêches : «Etre born again, c'est être sauvé! C'est quelque chose dont on est sûr et certain! »C'est là un argument de réassurance que ces migrantes converties accueillent favorablement, dans la mesure où elles connaissent un fort degré d'incertitude existentielle.

\section{La kafala, minoration et stratégie}

Au cours d'un terrain de recherche conduit à Beyrouth en septembre 2015, nous croisons par hasard le chemin d'Aimée Thirion, reporter d'image au journal français Libération et nous assistons à une scène particulière : Exaltaçion, accompagnée d'Aimée, est en discussion avec son employeur afin de renégocier les termes de son contrat de travail. La jeune femme voudrait pouvoir disposer de deux soirs par semaine et s'engage à dormir dans le studio de la missionnaire, attenant à l'église. La jeune migrante se tient la tête basse, dans une attitude humble. Son employeur lui donne le prénom de Nada, qui est celui d'une précédente domestique, au prétexte qu'il ne parvient pas à le prononcer convenablement, un déni d'identité qui participe d'une forme de déshumanisation ${ }^{7}$. Comme pour mieux attester physiquement de son invisibilisation, la migrante domestique porte son uniforme de domestique, alors qu'elle ne travaille pas. L'employeur insiste sur le fait qu'elle ne peut dormir que chez une femme célibataire et qu'il n'acceptera pas que la jeune femme ait une aventure amoureuse. "Vous comprenez... je ne pourrais pas accepter qu'elle euh... fréquente... c'est important pour elle et pour moi, vous comprenez ? » Aimée à qui le propos s'adresse hoche positivement la tête affirmant que la chose la préoccupe également. Cette volonté de neutralisation de la vie sexuelle par l'employeur renvoie à des représentations sociales et des dynamiques distinctives selon le genre, qui attribuent le plus souvent aux femmes un rôle actif en matière de séduction, source de suspicion ${ }^{8}$.

L'employeur affirme en outre se préoccuper du sort de son employée qu'il continue d'évoquer à la troisième personne, comme si elle ne se trouvait pas face à lui. "S'il lui arrive quelque chose, c'est ma responsabilité vous savez! » Un tel propos donne le sentiment qu'il évoque le cas d'une enfant à sa charge. Il convient ici de s'arrêter sur la notion de kafala. Selon Gilbert Beaugé, il s'agit d'un « système de gestion transitoire de la main-d'ceuvre et du capital ${ }^{9}$ ». Mais l'étymologie du mot nous en apprend davantage. Originellement, la kafala constitue un concept juridique de droit coranique qui désigne une mesure de recueil légal d'un enfant sans créer un lien de filiation, au contraire d'une adoption classique ${ }^{10}$. Dès lors, le parent qui adopte devient le protecteur et le garant de l'enfant. Il est aisé ici de saisir le mouvement qui prolonge ce système dans l'ordre des 
représentations, depuis l'espace privé de la famille jusqu'au domaine du travail, où une forme d'infantilisation et de déresponsabilisation des migrantes est à l'œuvre.

Judy est elle-même mère de famille mais ne voit ses enfants qu'une fois par an, lors des congés de Noël : "On se voit à Noël. Le reste du temps, on se parle beaucoup par téléphone, sur snapshat. On essaie de garder un lien étroit, mes fils m'envoient des mails, des photos, pour que je puisse partager en quelque sorte leur quotidien. Mais ce n'est pas pareil bien sûr que de se voir tous les jours. » Ici, tout concourt à perturber les normes de représentation de soi : comment peut-on concevoir de disposer du statut d'enfant lorsque l'on est une femme adulte? Comment peut-on vivre le fait d'être une mère séparée de ses enfants lorsque l'on en élève d'autres? Ce bouleversement des repères a d'importantes conséquences sur l'auto-représentation de soi. Dans des sociétés ou des groupes en proie à d'importantes mutations d'ordre sociopolitique ou un bouleversement des rôles sociaux, l'indétermination des rôles et des identités sont la cause d'un grand désarroi. La migrante, femme adulte et parfois mère de famille se voit assimilée par ses employeurs à une enfant qui ne saurait être maîtresse de ses décisions, et donc de son destin.

\section{Une échappatoire paradoxale}

Il est certain que la jeunesse a un caractère fluctuant selon les époques et les aires culturelles. Comme le soulignent Giovanni Levi et Jean-Claude Schmitt, la jeunesse est « un état provisoire que les individus ne font que traverser ${ }^{11}$ », un "fait social instable ». Mais, en dépit de sa relativité, la jeunesse est une temporalité balisée socialement de moments existentiels majeurs parmi lesquels la carrière professionnelle et la parentalité. Lorsque des jeunes femmes adultes ont un rôle social si peu défini, un risque de cassure apparaît dans la biographie qui nécessite un réajustement. Aimée nous confie avec émotion le cas d'une jeune femme qui s'est suicidée. Ce cas l'a d'autant plus désemparée que la jeune femme n'était pas maltraitée. La missionnaire a justement saisi que la solitude, l'invisibilisation et l'impossibilité à endosser un rôle social positif peut être la cause de ce désarroi extrême.

Ernest Kantorowicz explique que, face à un choc émotionnel lié, par exemple, à une inexistence sociale, qui se double ici d'un déracinement et d'une perte concomitante de ses repères, il est nécessaire de disposer d'un "équivalent émotionnel ${ }^{12}$ " sous peine de rupture. C'est là que l'insertion dans une communauté religieuse aux liens forts joue à plein. Le pentecôtisme en particulier, qui fait la part belle à l'émotivité et à l'affectivité peut agir positivement. Faute d'un tel principe, l'expérience brute de la violence, y compris symbolique, laisse l'individu démuni, et ne lui propose, pour tout instrument d'identification qu'une définition négative de luimême, une existence en creux, une abstraction.

En matière comportementale, il s'agit de modeler une employée selon des normes ultra-conservatrices adossées aux notions d'honneur et de respectabilité. L'employeur de Judy se veut le témoin de la bonne moralité de sa domestique dont elle surveille les fréquentations. En particulier, il s'agit de s'assurer que son employée n'a pas noué de relations amoureuses : «- Ta patronne sait que tu vas à l'église? - Oui bien sûr, elle sait. C'est une madame qui sait tout [elle rit]. Elle pense qu'elle doit tout savoir en tout cas. Elle pense que c'est bien d'aller à l'église, de ne pas fréquenter. Ça, c'est très important pour elle. »Ce capital réputation est une ressource précieuse qui permet à l'employeur de demeurer dans une posture d'autorité. Dans les négociations menées avec la "patronne ", la fréquentation de l'église est un argument fort pour solliciter une journée de repos. Fort habilement, Exaltaçion soutenue par Aimée peut ainsi retourner le moralisme de l'employeur contre lui-même et à son propre avantage, révélant une capacité réelle à jouer de stratégie. Dans l'entretien auquel nous avons assisté, l'employée aidée de la missionnaire obtient gain de cause.

Cela nous conduit à questionner les raisons pour lesquelles ces femmes migrantes se regroupent au 
sein d'églises. En effet, elles pourraient fort bien se réunir au sein d'associations de femmes ou d'un groupe culturel. Pour comprendre la raison de ce mode de socialisation et d'intégration, un extrait d'entretien avec Judy nous semble éclairant : « Les patrons sont contents que je passe mon temps dans l'église, ça les rassure. Ils voient du coup que je suis sérieuse. Parce qu'avant moi, ils avaient une fille de mauvaise vie qui sortait avec des hommes, le mauvais exemple quoi. Et tu vois, j’ai eu de la chance. Parce que certains patrons, quand ils voient ça, ils serrent la vis. ” Damien Mottier ${ }^{13}$ a démontré que, dans un pays comme la France où un grand nombre de migrants africains se regroupent pareillement dans des Églises évangéliques, ces derniers doivent affronter la suspicion de la société majoritaire, liée à un sentiment fort d'antireligionisme et Les témoignages une laïcité militante. Mais, au de ces jeunesfemmes Liban, tel n'est pas le cas. La qui s'ouvrent avant communauté religieuse est pleiou après chaque culte de nement légitime. La religion y leurs difficultés et de leurs bénéficie d'une dimension d'évisentiments s'expérimentent comme un sas de décompression, plus encore, une véritable thérapie. voient d'un bon œil leur fréquentation de l'église. C'est là une manière de réassurance. Les employeurs tendent à accorder plus aisément un congé dominical lorsque la jeune femme affirme qu'elle veut consacrer ce jour « off » à la fréquentation de l'église, censée constituer une marque d'honorabilité et de sérieux. L'église semble offrir à cet égard un modèle paradoxal : à la fois relais du contrôle social et normatif (défendant implicitement un certain rigorisme moral et sexuel) avalisé par les patrons (la " madame » et le « monsieur » étant également fort intéressés à la chasteté de leur employée, justifiant le contrôle exercé sur elle aux fins de " protection ») et, en même temps, un contre-pouvoir et un lieu de contournement de l'arbitraire de l'employeur.

\section{Le témoignage-thérapie}

En doctrine évangélique, le témoignage occupe une place centrale, dans la mesure où le croyant a le devoir d'attester de l'exemplarité de son parcours, souvent traversé d'épreuves, jusqu'au chemin de "vérité ». Il constitue aussi une manière de convaincre le converti potentiel de la justesse de ses choix spirituels. La valorisation de cet univers idéal où la parole est supposée libre de toute entrave est déjà mise en évidence par Yannick Fer et Joël Robbins dans d'autres espaces géographiques ${ }^{14}$.

À l'occasion des cultes et des nombreux autres espaces de rencontres (études bibliques, réunions de femmes, de jeunes etc.), le témoignage est omniprésent. Nous constatons que ces espaces de discussion fonctionnent comme une thérapie. Le fidèle se raconte, suscite l'émotion, le rire puis, au terme de son discours, il est souvent l'objet de manifestations d'amitié : étreintes, pression chaleureuse de la main sur l'épaule, grand sourire ou applaudissements nourris.

Le caractère effusionnel des manifestations affectives très marquées du protestantisme évangélique, notamment dans la mouvance pentecôtiste, joue ici sans doute un rôle attractif important. Les témoignages de ces jeunes femmes qui s'ouvrent avant ou après chaque culte de leurs difficultés et de leurs sentiments s'expérimentent comme un sas de décompression, plus encore, une véritable thérapie. Dans cette perspective, la conversion se fait réparatrice. La convertie trouve un moyen de positiver son expérience passée en la transformant en ressource pour soutenir à son tour d'autres migrantes «victimes » potentielles de leurs employeurs indélicats. Le cercle familial aboli par la situation de migration se recompose autour d'une famille chrétienne et est le lieu d'un nouveau départ, d'une nouvelle vie. La conversion est l'acte fort qui consacre la rupture et l'entrée dans une nouvelle ère existentielle. À bien y regarder, toutefois, cette prise en 
charge psychologique est fréquemment appuyée par un soutien effectif rendu possible par la forte cohésion de la communauté protestante évangélique et son fonctionnement en réseau.

\section{Une économie familiale repensée}

La plupart des migrantes envoient une grande partie de leurs revenus à leur famille demeurée dans leur pays d'origine. Dès lors, elles subissent une forme de double peine, dans la mesure où elles supportent un fardeau économique pesant, tout en subissant toujours un fort contrôle social qui les infantilise. En d'autres termes, ces femmes ont intégré le monde du travail sans profiter de ses avantages en termes d'indépendance financière et d'autonomie morale.

L'éthique évangélique qui intègre une dimension individualiste fondamentale permet d'abaisser le seuil de la culpabilité pour les individus qui voudraient s'en démettre. S'adonner aux plaisirs de la vie n'est plus taxé d'égoïsme superficiel, mais considéré comme une manière saine de rendre témoignage des gratifications accordées par Dieu en reconnaissance des efforts accomplis sur la voie du Seigneur. Par contraste, les demandes empressées des proches (surtout s'ils sont éloignés) en matière d'aide matérielle se voient stigmatisées. On songe d'abord à soi et aux très proches. C'est ainsi que Judy n'a plus donné suite aux demandes constantes d'aide financière émanant de sa cousine. Au lieu de cela, elle lui a conseillé de la rejoindre au Liban où sa parente est à présent employée elle-même comme domestique afin de subvenir à ses besoins et à ceux de ses proches. Comme l'affirme Élisabeth Dorier-Apprill: "La valorisation éthique du lien conjugal et du lien parents/enfants peut conduire, en condamnant comme "parasitisme" les "excès" des demandes d'aide dans le cadre lignager, à construire sur des bases évangéliques - un nouveau paradigme d'une solidarité "bien ordonnée", à la fois plus restreinte (compatible avec les besoins et même l'enrichissement de la famille nucléaire) et occasionnellement élargie (hors du lignage, en direction des "frères et sours en Christ") $)^{15}$. " C'est là une illustration d'un phénomène paradoxal déjà observé par Olivier Bobineau, selon lequel l'insistance sur la centralité de l'individu peut conduire à une remise en cause des solidarités familiales au profit d'autres liens affinitaires, reflet d'un «individualisme confinitaire ${ }^{16}$ ». Dès lors, l'église contribue à la diffusion de nouveaux modèles qui remettent en cause les valeurs hégémoniques de la société d'origine en replaçant l'individu au cœur de ses propres préoccupations.

\section{La réalisation de soi}

La mouvance évangélique promeut un modèle expérientiel religieux qui bouscule les identités et opère de profondes transformations des schèmes de perception de l'existence. Le mode de vie communautaire est recherché, la vie en groupe paraît plus exaltante, les liens disposent d'une plus forte intensité relationnelle, à travers la participation à un mouvement religieux doté de multiples liens directionnels. Il existe là une dimension importante d'intégration sociale que Daniel Gaxie a mis en exergue dans son étude des mouvements militants ${ }^{17}$.

Le motif instrumental ne saurait sans doute suffire à expliquer les motivations des converties, dans la mesure où l'insertion dans la communauté protestante évangélique réclame un investissement très lourd de ses membres, mobilisées dans de multiples activités déployées par l'église durant leur maigre temps libre ${ }^{18}$. Il n'en demeure pas moins qu'elles

15. Élisabeth Dorier-Apprill, Robert Ziavoula, "La diffusion de la culture évangélique en Afrique centrale. Théologie, éthique et réseaux ", in Hérodote, vol. 4, n 119, 2005, pp. 129-156. 16. Olivier Bobineau, "La troisième modernité ou l'individualisme confinitaire ", in Sociologies, Théories et Recherches, 2011. 17. Daniel Gaxie, La démocratie représentative, Paris, LGDJ / Montchrestien, 2000. 
trouvent dans leur nouvelle situation des avantages concrets non négligeables, rendus possibles par le fonctionnement en réseau de l'église.

Ainsi, dans une société ultra libérale comme le Liban où les soins de santé sont fort coûteux, la communauté protestante intègre en son sein des médecins, des avocats, et diverses professions qui proposent gracieusement leurs services. Judy, qui rencontre des problèmes de santé, trouve dans la prière une consolation pour ses peines. Mais elle recourt également aux services gratuits d'un médecin évangélique : «Il suffit de lire la Bible et on trouve la solution. En plus, on est solidaires concrètement. Si une fille a un problème d'argent, si elle a un problème avec un employeur, on l'aide. On a des services et des avocats. On a aussi un médecin où

La mouvance évangélique promeut un modèle expérientiel religieux qui

bouscule les identités et opère de profondes

transformations des schèmes de perception de l'existence. les filles vont gratuitement. Le médecin que je vois est un Libanais évangélique, il ne me fait pas payer. »

Les migrantes ont, en outre, la possibilité de suivre gratuitement des cours d'arabe et d'anglais à Koreitem. De ce fait, on voit que l'intégration dans un réseau évangélique ouvre un champ large d'opportunités pour les jeunes femmes. Plus largement, il est possible de mobiliser ici la notion de « capabilité » analysée par Amartya Sen ${ }^{19}$ qui désigne la capacité des individus à agir, à divers degrés, comme des acteurs sociaux de façon responsable et indépendante en dépit des pesanteurs de l'organisation sociale.

\section{Conclusion}

La conversion ne conduit pas à une transformation radicale des existences des migrantes employées comme domestiques, mais elle autorise un processus de réajustement en forme d'accommodement raisonnable qui affecte l'existence de façon discrète mais décisive.

L'église, lieu de rassemblement de groupes de femmes solidaires, devient un espace de résistance à un ordre social dominé par les hommes et par les employeurs autochtones. Elle est le lieu à la fois clos et intime qui permet aux femmes de connaître ce que Françoise Héritier appelle « le rêve de rester entre soi ${ }^{20}$ ». Dans ces rendez-vous presque exclusivement féminins, une abolition des préjugés s'opère. Là encore, l'église est un espace de transgression propice à une indifférenciation apparente des rôles sociaux attestée par le discours : "Nous sommes tous enfants de Dieu ». Une posture qui affirme l'égalité entre tous.

Cependant, il convient de nuancer ce constat. En l'absence d'une évolution du statut juridique des migrantes domestiques, les opportunités qu'elles trouvent dans l'église semblent bien souvent fonctionner comme des techniques de contournement ou d'adoucissement des contraintes objectives auxquelles elles sont confrontées. Préalable indispensable à une amélioration du sort de ces employées domestiques, la réforme de la kafala, en vue d'une meilleure codification du statut des travailleuses domestiques migrantes se fait toujours attendre. 